\title{
Enhancement of DNA cancer vaccine efficacy by combination with anti-angiogenesis in regression of established subcutaneous B16 melanoma
}

\author{
RAY CHUN-FAI CHAN ${ }^{1}$, BENJAMIN GUTIERREZ ${ }^{1}$, THOMAS E. ICHIM ${ }^{2}$ and FENG LIN ${ }^{1}$ \\ ${ }^{1}$ Inovio Biomedical Corporation, 11494 Sorrento Valley Road, San Diego, CA 92121; \\ ${ }^{2}$ Medistem Inc, 9255 Towne Centre Drive, Suite 450, San Diego, CA 92122, USA
}

Received May 28, 2009; Accepted July 10, 2009

DOI: $10.3892 /$ or_00000555

\begin{abstract}
Immunotherapy of cancer offers great promise, however translation into human studies has yielded relatively poor results to date. The concept of combining cancer vaccination with angiogenesis inhibition is appealing, due to favorable safety profile of both approaches, as well as possible biological synergies. Here we studied the anti-tumor effects of combining plasmid DNA (pDNA) vaccination and anti-angiogenesis in B16F10 murine model. By using electroporation-mediated gene/ pDNA delivery, the anti-tumor efficacy of vaccination with pDNAs encoding gp100, TRP2 and Ii-PADRE was facilitated by administration of soluble form of EphB4 fused with human serum albumin (sEphB4-HSA), or by codelivery of pDNAs encoding Angiostatin and/or Endostatin. In an optimized administration protocol, melanoma vaccination together with intratumoral delivery of pDNAs encoding Angiostatin and Endostatin resulted in 57\% tumor-free survival over 90 days after challenge. These data support the general concept that suppression of angiogenesis may allow for enhanced efficacy of anti-tumor immunity, suggesting the synergetic effects of therapeutic pDNA vaccination and angiogenesis inhibition in cancer therapy.
\end{abstract}

\section{Introduction}

Angiogenesis is essential for the growth, establishment and even metastasis of tumors (1). Angiogenesis is not present in non-malignant adult tissues except during wound healing

Correspondence to: Dr Feng Lin, Department of Research and Development, Inovio Biomedical Corporation, 11494 Sorrento Valley Road, San Diego, CA 92121-1318, USA

E-mail: flin@inovio.com

Key words: melanoma, DNA vaccine, electroporation, antiangiogenesis or in the corpus luteum and endometrium. In tumor tissue, angiogenesis is however stimulated through the secretion of vascular endothelial cell growth factors (VEGFs) and fibroblast growth factors (FGFs) (2). The concept of targeting the endothelium as a means of 'starving' the tumor is attractive since unlike the tumor itself, the endothelium is host derived and therefore has a lower mutation rate and lower possibility of drug resistance. Extensive studies by many investigators using angiogenesis inhibitors show suppression of tumor growth in animal models (3-6). However, the use of these inhibitors has only produced limited success in the complete regression of established tumors (3-9). Thus, improved strategies where angiogenesis inhibitors are combined with alternative treatments to improve efficacy is warranted.

Recent advances in the molecular identification of melanoma-specific antigens have given a significant boost to the study of novel vaccines for melanoma (10). Plasmid DNA (pDNA) vaccines are well suited to deliver cancer antigens since this modality effectively induces cellular immune responses similar to that induced by live, attenuated viral vaccines. Recently, in vivo electroporation has emerged as a potent method to increase the immunogenicity of pDNA vaccines in larger animal models as well as humans (11-15). Electroporation leads to enhanced antigen expression and an adjuvant effect due to local tissue damage and this combination increases immune responses, especially, enabling effective generation of cytotoxic $\mathrm{T}$ lymphocytes (CTLs) $(16,17)$. In the case of cancer vaccines, the elicited CTLs however require entry into neoplastic tissue to mediate anti-tumor effects $(18,19)$. This process is often ineffective in tumors, partially because of vessel wall abnormalities associated with hyper-angiogenesis (2). Therefore, the combination of cancer vaccine with antiangiogenesis factors may produce synergistic anti-tumor effects in cancer therapy.

Here we investigated the synergetic anti-tumor effects of vaccination with pDNAs encoding gp100, TRP2 and IiPADRE combined with systemic inhibition of angiogenesis by an EphrinB2 antagonist comprised of an extracellular domain of EphB4 fused with human serum albumin (sEphB4-HSA), or by delivery of pDNAs encoding Angiostatin and/or Endostatin in a highly aggressive B16F10 
mouse melanoma model. All the pDNAs were delivered by in vivo electroporation.

\section{Materials and methods}

Construction of plasmids. Genes encoding human glycoprotein 100 (hgp100), human tyrosinase-related protein 2 (hTRP2), murine gp100 (mgp100), murine TRP2 (mTRP2), murine invariant chain (Ii) with CLIP replaced by pan-DR-epitope (Ii-PADRE) (20), murine genes Endostatin and Angiostatin were codon-optimized and synthesized by GeneArt (Regensburg, Germany). The genes were sequenced and subcloned into the proprietary clinically acceptable vector, pMB75.6 (Inovio B.C., San Diego, CA). An overview of the constructed plasmids DNA is shown in Fig. 1. Plasmids were grown in Escherichia coli strain TOP10 (Invitrogen, Carlsbad, CA) and purified using EndoFree plasmid Maxi kit according to the manufacturer's descriptions (Qiagen, Valencia, CA). The purified plasmid DNAs were dissolved in water and stored at $-20^{\circ} \mathrm{C}$.

Mice. Female C57BL/6 mice (6-8 weeks old) $\left(\mathrm{H}-2^{\mathrm{b}}\right)$ were purchased from Charles River Laboratories (San Diego, CA). All animal experiments were conducted at the Bioquant Inc. (San Diego, CA) in adherence to the standards of Institutional Animal Care and Use Committee (IACUC) for the care and use of laboratory animals. All subcutaneous injections were performed on mice anesthetized with methoxyflurane.

Cell lines. B16F10, a spontaneous murine melanoma cell line of C57BL/6 $\left(\mathrm{H}-2^{\mathrm{b}}\right)$ origin, expressing mouse gp100 and TRP2 was obtained from ATCC. Cells were cultured in Dulbecco's Modified Eagle's Medium (DMEM) supplemented with $10 \%$ fetal bovine serum, $2 \mathrm{mmol} / \mathrm{l} \mathrm{L}$-glutamine, $100 \mathrm{IU} / \mathrm{ml}$ penicillin and $100 \mu \mathrm{g} / \mathrm{ml}$ streptomycin at $37^{\circ} \mathrm{C}$ in a humidified $5 \% \mathrm{CO}_{2}$ incubator.

EphrinB2 antagonist (sEphB4-HSA). The soluble form of EphB4 (sEphB4) fusion protein consisting of the extracellular domain of the receptor fused to human serum albumin (sEphB4-HSA) was generated and provided by VasGene Therapeutics, Inc. (Los Angeles, CA) $(8,21,22)$. Mice were injected intraperitoneally (i.p.) with $20 \mathrm{mg} / \mathrm{kg}$ sEphB4-HSA diluted in $100 \mu$ l PBS three times a week for two weeks starting on day 10 after B16F10 challenge.

Immunization and electroporation. Mice were immunized intramuscularly (i.m.) with $10 \mu \mathrm{g}$ of each plasmid DNA in $40 \mu 1$ saline. Empty vector was added to keep the DNA amount equal for immunization in each group. Plasmid injection was followed immediately by i.m. electroporation (125 V/cm, 2 pulses of $60 \mathrm{~ms}$ each) using Elgen 1000 with 2 needles/electrodes (Inovio B.C.). For intratumoral (i.t.) electroporation, $10 \mu \mathrm{g}$ of each plasmid encoding murine Endostatin/Angiostatin or empty vector were injected into tumor at day 10 after B16F10 challenge when the tumor size reached $20-50 \mathrm{~mm}^{3}$. Immediately electroporation was conducted using a four-needle electrode array that was inserted into the tissue around the tumor (the injection site). The

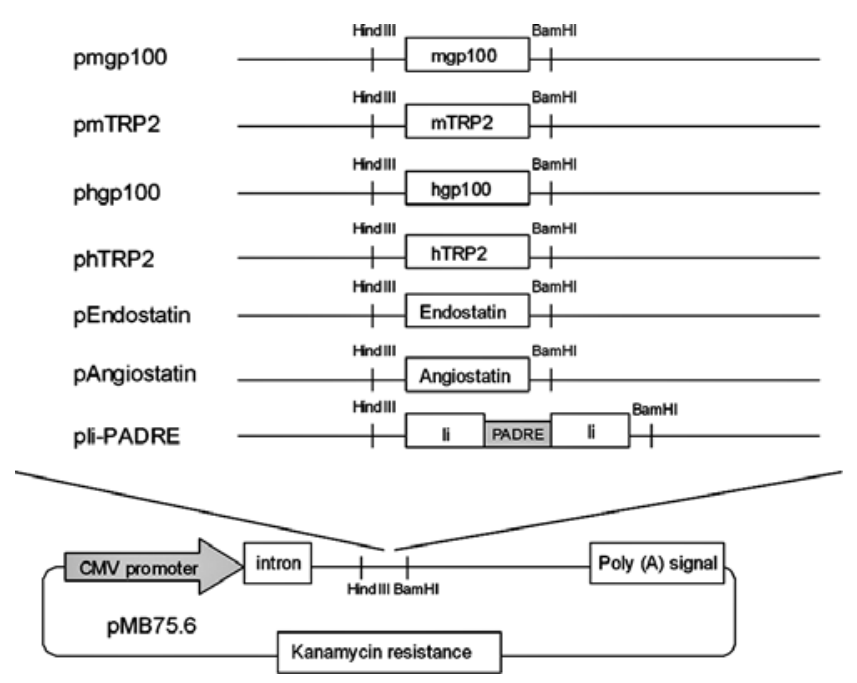

Figure 1. Schematic diagram of the DNA constructs. The murine gp100 (mgp100), mTRP2, human gp100 (hgp100), hTRP2, murine genes Endostatin and Angiostatin were cloned into the pMB75.6 vector under the CMV promoter control as indicated. For pIi-PADRE, the murine invariant chain molecule with the CLIP region was replaced by PADRE sequence.

parameters, 4 pulses of $20 \mathrm{~ms}$ length each at $400 \mathrm{~V} / \mathrm{cm}$, were applied using Elgen 1000.

Therapeutic study. B16F10 cells $\left(2 \times 10^{5}\right)$ were injected into the left flank and grown into a visible tumor after 5 days. Mice were immunized with plasmid DNAs on day 6, 13, 20 and 27. In some experiments, mice were injected i.p. with $100 \mu 1$ of sEphB4-HSA or PBS 3 times a week for two weeks, starting on day 10 after B16F10 challenge. Tumor volumes were determined 3 times a week using a digital caliper by measuring the longest diameter (a) and shortest width (b) of the tumor. The tumor volume was calculated by formula $\mathrm{V}\left(\mathrm{mm}^{3}\right)=0.5$ $\mathrm{x} \mathrm{ab}^{2}$. For humane reasons, animals were euthanized when the tumor reached $>1500 \mathrm{~mm}^{3}$.

Statistical analyses. The statistical significance between experimental groups was determined by Student's t-test. The results were considered to be significant when the P-value was $<0.05$. The Kaplan-Meier test was used to analyze different group survival rates.

\section{Results}

Inhibition of angiogenesis by sEphB4-HSA enhances DNA vaccine efficacy against B16 tumor. To elucidate whether the efficacy of cancer DNA vaccines can be enhanced by suppressing tumor angiogenesis, we combined a pDNA vaccine delivered by in vivo electroporation with systemic administration of sEphB4-HSA, a factor that blocks the interaction between EphrinB2 and EphB4 resulting in suppression of angiogenesis in tumor. DNA vaccination was performed in C57BL/6 mice with plasmids encoding hgp 100 , hTRP2 and Ii-PADRE, which have been shown to induce anti-tumor effects against B16F10 tumor (unpublished data). In the prophylactic study, we observed that mice vaccinated alone with phgp100, phTRP2 and pIi-PADRE generated a 
A
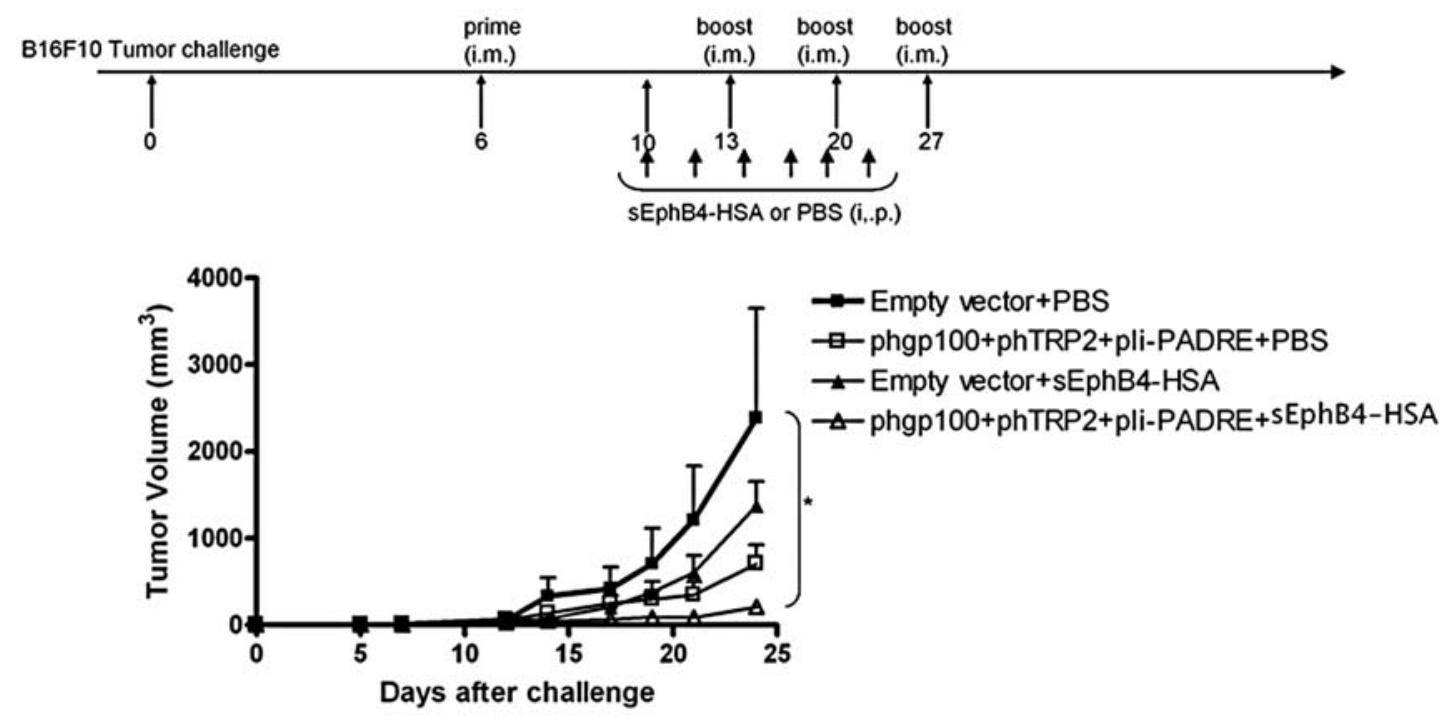

B
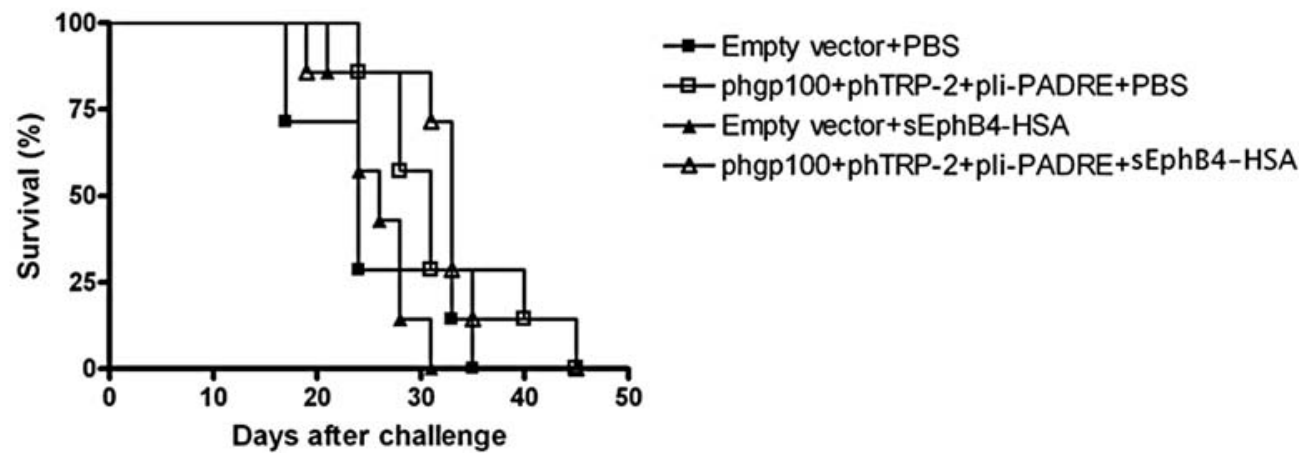

$\triangle$ phgp100+phTRP-2+pli-PADRE+sEphB4-HSA

Figure 2. Anti-tumor effect of melanoma DNA vaccine was enhanced by sEphB4-HSA. (A) The immunization scheme shows time points of vaccination and B16F10 tumor challenge as indicated by arrows. sEphB4-HSA or PBS (100 $\mu 1)$ were given intraperitoneally (i.p.) starting at day 10 three times a week for 2 weeks and DNA vaccine (phgp100+phTRP2+Ii-PADRE or empty vector) was injected intramuscularly (i.m.) followed by electroporation. For boosting on day 13 and 27, the plasmids encoding hgp100 and hTRP2 were changed to mgp100 and mTRP2, respectively. Each point represents mean of tumor volume with SEM bars of animals of each group $(\mathrm{n}=7)$. Curves of the groups were significant different when $\mathrm{P}<0.05$. (B) Survival analysis of mice in the therapeutic model.

significant anti-tumor effect against B16F10 tumor as compared to the mice immunized with empty vector alone (Fig. 2A). In confirmation of previous results obtained from other tumor models by Scehnet et al (8), administration of sEphB4-HSA alone also resulted in significant suppression of B16 tumor growth (Fig. 2A). Intriguingly, the treatment combining phgp100, phTRP2 and pIi-PADRE with sEphB4HSA greatly increased the anti-tumor effects (Fig. 2A), suggesting that blocking of angiogenesis may slow down tumor growth thereby enhancing the DNA cancer vaccine efficacy. There was no significant difference in overall survival rates between the vaccinated groups with or without sEphB4HSA, although a trend towards improved survival for the later group at earlier time points was observed (Fig. 2B). It is however plausible that continued administration of sEphB4HSA beyond day 21 could further improve the survival in this group.

Intramuscular co-delivery of plasmids encoding Endostatin and/or Angiostatin enhances melanoma DNA vaccine efficacy against B16 tumor. To assess whether potentiation of the DNA vaccine efficacy was specific to EphrinB2 antagonism, or if the observed synergy is a general property associated with inhibition of angiogenesis, we further combined the vaccine with the angiogenesis inhibitors, Endostatin and Angiostatin $(5,6)$ in a therapeutic study. Using in vivo electroporation, the mixture of plasmids encoding Endostatin and/or Angiostatin with the melanoma vaccine was co-delivered into gastrocnemius muscle of tumor-bearing mice. Mice vaccinated with the melanoma vaccine (phgp100+phTRP2+pIi-PADRE) displayed a steeper tumor growth curve as previously observed (Fig. 3A vs. 2A). The growth discrepancy was probably caused by variation of tumor implantation that we have previously observed in our studies. Importantly, the addition of plasmid encoding either Endostatin or Angiostatin enhanced the anti-tumor response of the melanoma vaccine (Fig. 3A). Furthermore, combination of the melanoma vaccine with plasmids encoding Endostatin and Angiostatin could exert stronger anti-tumor effect and prolonged the survival significantly, suggesting that targeting different angiogenesis pathways simultaneously may provide additional therapeutic benefits in this tumor model (Fig. 3A and B). 
A
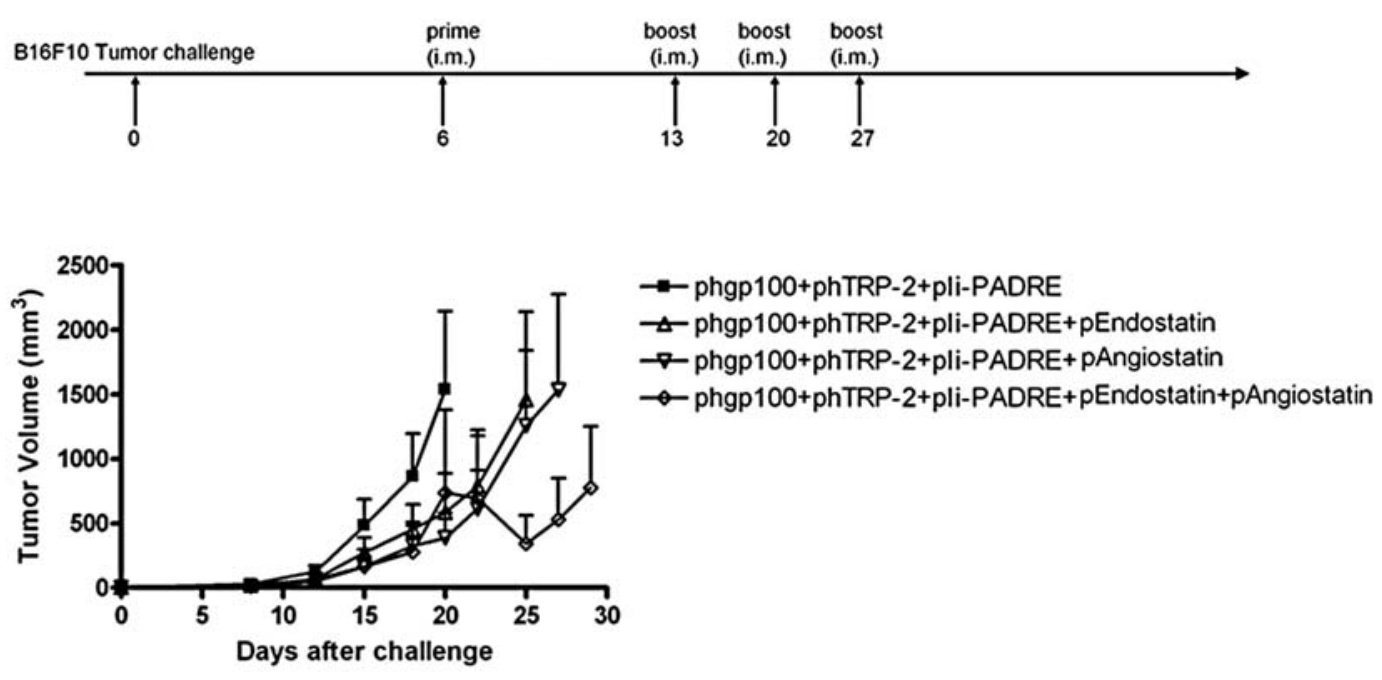

B

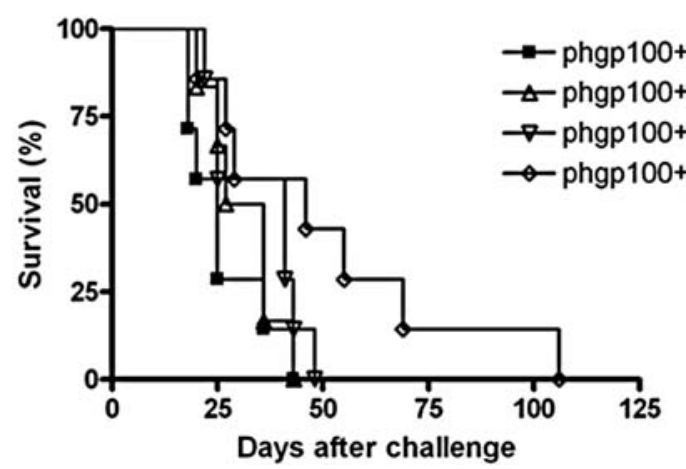

Figure 3. Anti-tumor effect of melanoma DNA vaccine enhanced by i.m. delivered Endostatin and/or Angiostatin plasmids. (A) The immunization scheme indicates the days of vaccination and B16F10 tumor challenge by arrows. DNA (phgp100+phTRP2+Ii-PADRE or empty vector) together with pEndostatin and/or pAngiostatin were delivered by i.m. electroporation. For boosting on day 13 and 27, the plasmids encoding hgp100 and hTRP2 were changed to mgp100 and mTRP2, respectively. Each point represents mean of tumor volume with SEM bars of animals of each group (n=7). Curves of the groups were significant different when $\mathrm{P}<0.05$. (B) Survival analysis of mice immunized with melanoma vaccine and anti-angiogenic factors (i.m.) by electroporation in this therapeutic model. ( $\square$ vs. $\diamond, \mathrm{P}=0.023)$.

Intratumoral delivery of plasmids encoding Endostatin and Angiostatin further enhances melanoma DNA vaccine efficacy and increases tumor-free survival. Intramuscular (i.m.) codelivery of plasmids encoding Endostatin and Angiostatin with melanoma vaccine increased the overall survival. However, this combined treatment still failed to cause complete tumor regression and provide long-term tumor-free survival (Fig. 3B). It might be attributed to limited plasma levels of Endostatin and Angiostatin generated by the i.m. delivery. Intratumoral (i.t.) delivery of plasmid DNA by electroporation may result in higher expression levels in the tumor site (unpublished data). We therefore explored i.t. delivery of both Endostatin and Angiostatin plasmids as a way to provide more efficient inhibition of tumor angiogenesis in another therapeutic study. Mixture of Endostatin and Angiostatin plasmids was i.t. delivered by in vivo electroporation, empty vector was used as control. Meanwhile, melanoma plasmid vaccine was delivered by i.m. electroporation. As shown in Fig. 4A, i.t. delivery of Endostatin and Angiostatin plasmids alone had poor effect on suppression of the B16 tumor growth, suggesting that even i.t. delivery did not give rise to expression of therapeutic levels. Nevertheless, when combined with i.m. delivery of melanoma vaccine, i.t. delivery of Endostatin and Angiostatin plasmids significantly enhanced the anti-tumor effects of vaccination (Fig. 4A) and generated 57\% tumor-free survival over 90 days after challenge compared to $28 \%$ tumor-free survival with melanoma DNA vaccination alone (Fig. 4B), suggesting that i.t. administration of Endostatin and Angiostatin plasmids could further enhance the melanoma DNA vaccine potency against B16 tumor. It was noteworthy that mice received melanoma DNA vaccination with i.t. delivery of empty vector showed not only significant anti-tumor responses as compared to mice administered empty vector (Fig. 4A) but also tumor-free survival (Fig. 4B), although the survival was low it could not be observed in Figs. 2 and 3, suggesting that i.t. injection of empty vector followed by electroporation might lead to favorable changes in the tumor microenvironment enhancing the effect of antigen-specific CTLs induced by the melanoma vaccine (Fig. 4B).

\section{Discussion}

Here we report that suppression of angiogenesis by three different anti-angiogenesis factors or combinations thereof 
A
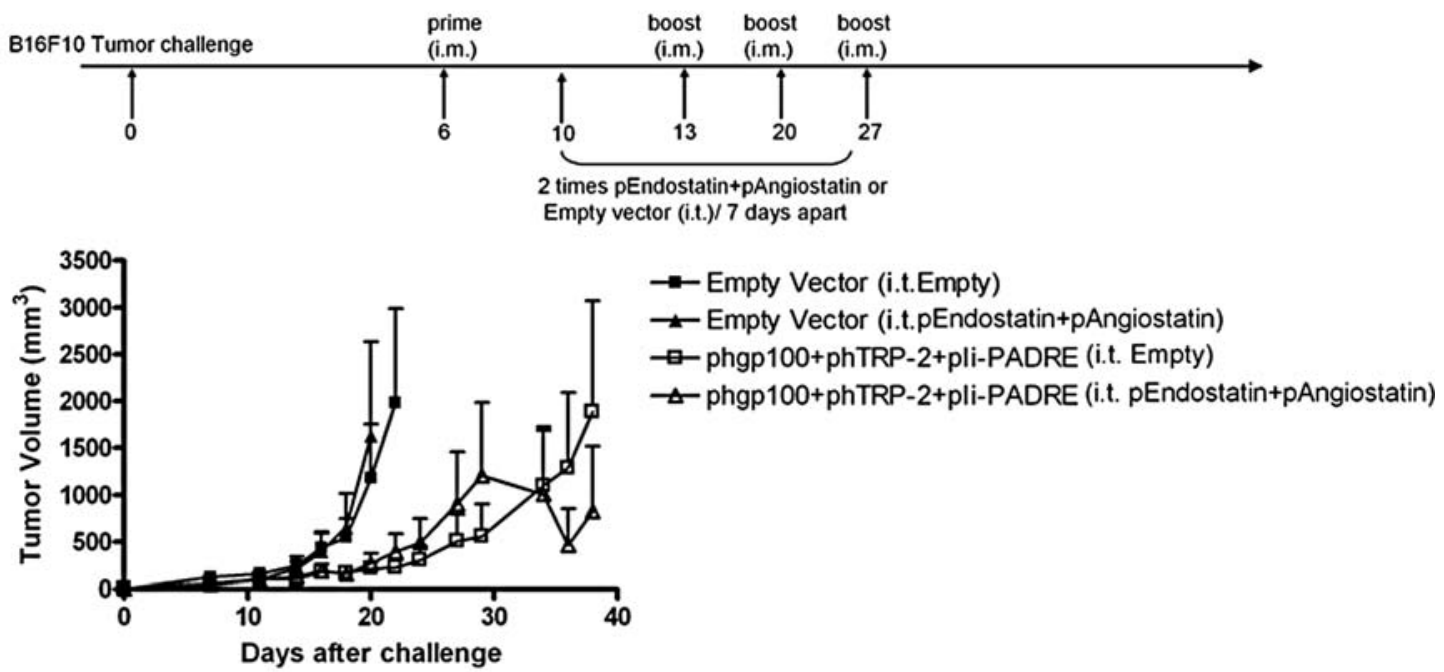

B

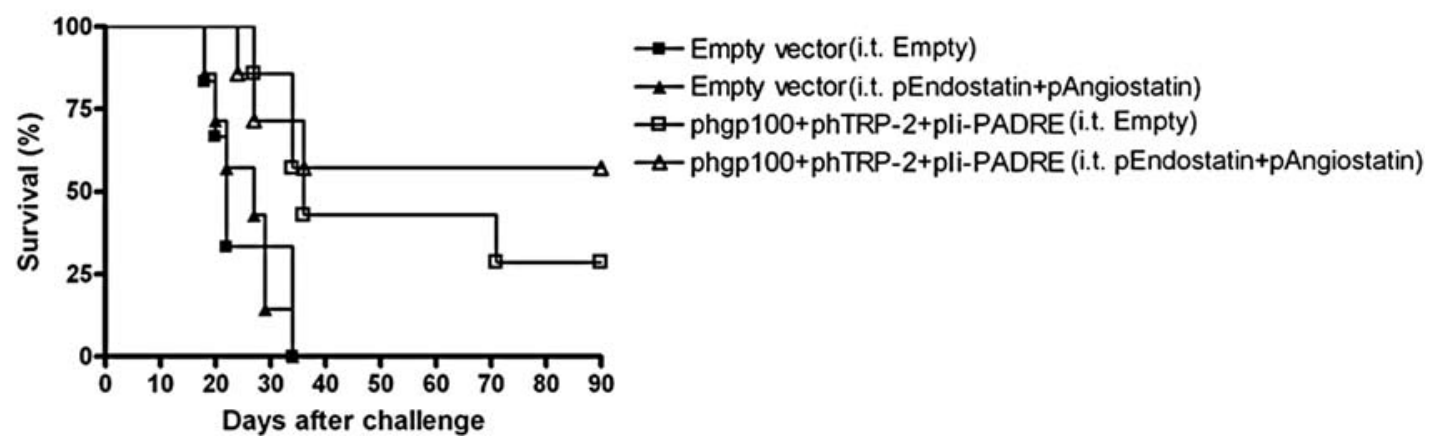

Figure 4. Anti-tumor effect of melanoma DNA vaccine enhanced by intratumorally (i.t.) delivered Endostatin and Angiostatin plasmids. (A) The immunization scheme indicates the days of vaccination and B16F10 tumor challenge by arrows. At day 6 after challenge, mice were immunized with plasmids encoding hgp100, hTRP2 and Ii-PADRE or empty vector as control i.m. followed by electroporation. For i.t. electroporation-mediated delivery, $10 \mu$ g of each plasmid encoding murine Endostatin or Angiostatin or empty vector were injected into the tumor followed by i.t. electroporation once the tumor size reached $20-50 \mathrm{~mm}^{3}$ and then repeated the treatment once in 7 days apart. For boosting on day 13 and 27, the plasmids encoding hgp 100 and hTRP 2 were changed to mgp100 and mTRP2, respectively. Each point represents mean of tumor volume with SEM bars of animals of each group ( $\mathrm{n}=7$ ). (B) Survival analysis of mice immunized with melanoma vaccine and anti-angiogenic factors (i.t.) by electroporation in the therapeutic model $(\mathbf{\square s} . \square, \mathrm{P}=0.0098 ; \boldsymbol{\nabla}$ vs. $\triangle, \mathrm{P}=0.0082)$.

potentiates the efficacy of a pDNA cancer vaccine expressing the melanoma associated antigens gp100 and TRP2. Following i.t. delivery of anti-angiogenesis genes combined with i.m. pDNA vaccination, we observed regression of established B16F10 tumor $\left(>50 \mathrm{~mm}^{3}\right)$ in our therapeutic study. To our knowledge, this is the first study to show $>50 \%$ tumor-free survival for over 90 days after challenge.

In our previous studies we have found that immunization with pDNAs encoding gp100, TRP2 and Ii-PADRE could elicit strong cytotoxic T lymphocyte (CTL) response in vitro and suppress B16F10 tumor growth in mouse model (unpublished data). However therapeutic immunization with the pDNA vaccine could not eradicate established tumor completely. One possibility is that the growing B16F10 tumor secretes immunosuppressive cytokines such as VEGF, IL-10 and TGF- $\beta$ which prevent the activated CTLs from entering into the tumor $(23,24)$. Here we find that the efficacy of vaccination can be substantially enhanced by blocking angiogenesis in tumor. By obstructing angiogenesis in tumors, endothelial cells in the tumor may be reverted to anergy and increase leukocyte infiltration (18). In addition, reducing blood and nutrient supply will slow down tumor growth and provide more time for the vaccine to stimulate effector cells against the tumor (2). This may be important especially for immunotherapy against fast growing tumors where the window of opportunity for effective treatment is limited. Other studies have demonstrated that Angiostatin and Endostatin can slow down tumor growth, prevent metastasis and prolong survival $(3,25)$. However, anti-angiogenesis factors did not stimulate specific immune response against the tumor and failed to eradicate already establish tumor completely $(3,25)$. The data presented here demonstrate that combining anti-angiogenesis factors with a therapeutic cancer vaccine may overcome this limitation and generate potent synergistic anti-tumor effects.

To inhibit tumor angiogenesis, we used the EphrinB2 antagonist (sEphB4-HSA), as well as Endostatin and Angiostatin. The ability of these factors to suppress angiogenesis in tumor have been well established $(5,6,8)$. Each of these angiogenesis inhibitors could enhance the efficacy of the tested melanoma cancer vaccine. sEphB4-HSA was delivered as a purified protein (generated by VasGene Therapeutics Inc). It has been proven not only to function as 
an EphrinB2 antagonist, but also to inhibit the activity of several growth factors including VEGF and bFGF (8). Theoretically, genetically encoded sEphB4-HSA may offer advantages with regards to cost and practicality since long-term expression may ablate the need for frequent administration. However, technical constrains precluded us from performing studies using pDNA encoded sEphB4-HSA. We therefore tested the alternative approach of delivering the genes encoding Endostatin and Angiostatin using in vivo electroporation. Our results validated the feasibility and efficacy of this approach, and clearly demonstrated that administering both Endostatin and Angiostatin genes was more effective than delivery of either gene alone (Fig. 3). The results are in line with those obtained by Scehnet and co-workers (8) who demonstrated that synergistic anti-tumor effects could be reached by combining two different anti-angiogenesis factors, Avastin and sEphB4-HSA. Angiogenesis is an extremely complex process involving multiple pathways and with a great deal of redundancy. Angiostatin inhibits endothelial cells migration and proliferation most likely through its ability to bind cell surface ATP synthase (26), while Endostatin seems to inhibit endothelial cell functions by binding to $\alpha 5 \beta 1$ integrins, attenuation of VEGF receptor signaling and inhibition of cyclin D1 (27). While the tumor may more easily escape the effect of either factor alone by up regulating alternative signaling pathway(s) this is more difficult when targeting two different pathways simultaneously. Further study needs to be done to address the real clinical benefits of therapies applying multiple anti-angiogenesis factors.

Our results indicate that i.t. delivery of angiostatic genes is more effective than i.m. delivery of the same constructs (Figs. 3 and 4). In previous studies we have found that electroporation-mediated i.t. delivery of IL-12 gene produced up to 20 times higher IL-12 in tumor as compared to i.m. electroporation delivery (unpublished results). Similar results have been observed using other genes including IL-15 and secreted human placental alkaline phosphatase (SEAP) (unpublished results). In the study described in Fig.3, however, the plasmids encoding Endostatin and Angiostatin were co-delivered with the melanoma DNA vaccine at the same injection site. Cytotoxic T-cell responses elicited by the vaccination could target the antigen-producing muscle cells, resulting in pre-mature elimination of expression of Endostatin and Angiostatin. Further experiments with separate delivery of pDNA vaccine and anti-angiogenesis factors in larger animal models are needed to address issues regarding dosing and utility.

In conclusion, our results demonstrate that combining electroporation-enhanced delivery of pDNA vaccine expressing multiple melanoma antigens with angiostatic treatment can eradicate established B16F10 tumor in mice. These findings warrant further investigation into combination therapies to increase effectiveness of therapeutic vaccines against malignant melanoma and other cancers.

\section{Acknowledgements}

We thank Dr Eric Sjoberg at VasGene Therapeutics Inc. for providing purified sEphB4-HSA and technical advice on using this protein.

\section{References}

1. Folkman J: Angiogenesis in cancer, vascular, rheumatoid and other disease. Nat Med 1: 27-31, 1995.

2. Griffioen AW: Anti-angiogenesis: making the tumor vulnerable to the immune system. Cancer Immunol Immunother 57: 1553-1558, 2008.

3. Cao Y, O'Reilly MS, Marshall B, Flynn E, Ji RW and Folkman J: Expression of angiostatin cDNA in a murine fibrosarcoma suppresses primary tumor growth and produces long-term dormancy of metastases. J Clin Invest 101: 1055-1063, 1998.

4. Niethammer AG, Xiang R, Becker JC, Wodrich H, Pertl U, Karsten G, Eliceiri BP and Reisfeld RA: A DNA vaccine against VEGF receptor 2 prevents effective angiogenesis and inhibits tumor growth. Nat Med 8: 1369-1375, 2002.

5. O'Reilly MS, Boehm T, Shing Y, Fukai N, Vasios G, Lane WS, Flynn E, Birkhead JR, Olsen BR and Folkman J: Endostatin: an endogenous inhibitor of angiogenesis and tumor growth. Cell 88: 277-285, 1997.

6. O'Reilly MS, Holmgren L, Shing Y, Chen C, Rosenthal RA, Moses M, Lane WS, Cao Y, Sage EH and Folkman J: Angiostatin: a novel angiogenesis inhibitor that mediates the suppression of metastases by a Lewis lung carcinoma. Cell 79: 315-328, 1994.

7. Mullen JT, Donahue JM, Chandrasekhar S, Yoon SS, Liu W, Ellis LM, Nakamura H, Kasuya H, Pawlik TM and Tanabe KK: Oncolysis by viral replication and inhibition of angiogenesis by a replication-conditional herpes simplex virus that expresses mouse Endostatin. Cancer 101: 869-877, 2004.

8. Scehnet JS, Ley EJ, Krasnoperov V, Liu R, Manchanda PK, Sjoberg E, Kostecke AP, Gupta S, Kumar SR and Gill PS: The role of Ephs, Ephrins, and growth factors in Kaposi sarcoma and implications of EphrinB2 blockade. Blood 113: 254-263, 2009.

9. Xu YF, Zhu LP, Hu B, Rong ZG, Zhu HW, Xu JM, Wu ZW, Wang JJ and Xu GX: A quantitative method for measuring the anti-tumor potency of recombinant human Endostatin in vivo. Eur J Pharmacol 564: 1-6, 2007.

10. Rosenberg SA: Development of cancer immunotherapies based on identification of the genes encoding cancer regression antigens. J Natl Cancer Inst 88: 1635-1644, 1996.

11. Daud AI, DeConti RC, Andrews S, Urbas P, Riker AI, Sondak VK, Munster PN, Sullivan DM, Ugen KE, Messina JL and Heller R: Phase I trial of interleukin-12 plasmid electroporation in patients with metastatic melanoma. J Clin Oncol 26: 5896-5903, 2008

12. Heller R, Schultz J, Lucas ML, Jaroszeski MJ, Heller LC, Gilbert RA, Moelling K and Nicolau C: Intradermal delivery of interleukin-12 plasmid DNA by in vivo electroporation. DNA Cell Biol 20: 21-26, 2001

13. Isaka $Y$ and Imai E: Electroporation-mediated gene therapy. Expert Opin Drug Deliv 4: 561-571, 2007.

14. Lucas ML, Heller L, Coppola D and Heller R: IL-12 plasmid delivery by in vivo electroporation for the successful treatment of established subcutaneous B16.F10 melanoma. Mol Ther 5: 668-675, 2002.

15. Luxembourg A, Evans CF and Hannaman D: Electroporationbased DNA immunisation: translation to the clinic. Expert Opin Biol Ther 7: 1647-1664, 2007.

16. Ahlen G, Soderholm J, Tjelle T, Kjeken R, Frelin L, Hoglund U, Blomberg P, Fons M, Mathiesen I and Sallberg M: In vivo electroporation enhances the immunogenicity of hepatitis $\mathrm{C}$ virus nonstructural 3/4A DNA by increased local DNA uptake, protein expression, inflammation, and infiltration of $\mathrm{CD}^{+} \mathrm{T}$ cells. J Immunol 179: 4741-4753, 2007.

17. Ledwith BJ, Manam S, Troilo PJ, Barnum AB, Pauley CJ, Griffiths TG, Harper LB, Beare CM, Bagdon WJ and Nichols WW: Plasmid DNA vaccines: investigation of integration into host cellular DNA following intramuscular injection in mice. Intervirology 43: 258-272, 2000.

18. Dirkx AE, oude Egbrink MG, Castermans K, van der Schaft DW, Thijssen VL, Dings RP, Kwee L, Mayo KH, Wagstaff J, Bouma-ter Steege JC and Griffioen AW: Anti-angiogenesis therapy can overcome endothelial cell anergy and promote leukocyte-endothelium interactions and infiltration in tumors. FASEB J 20: 621-630, 2006.

19. Dirkx AE, Oude Egbrink MG, Wagstaff J and Griffioen AW: Monocyte/macrophage infiltration in tumors: modulators of angiogenesis. J Leukoc Biol 80: 1183-1196, 2006. 
20. Hung CF, Tsai YC, He L and Wu TC: DNA vaccines encoding Ii-PADRE generates potent PADRE-specific CD4 ${ }^{+}$T-cell immune responses and enhances vaccine potency. Mol Ther 15: 1211-1219, 2007.

21. Liu W, Ahmad SA, Jung YD, Reinmuth N, Fan F, Bucana CD and Ellis LM: Coexpression of ephrin-Bs and their receptors in colon carcinoma. Cancer 94: 934-939, 2002.

22. Nakamoto $M$ and Bergemann AD: Diverse roles for the Eph family of receptor tyrosine kinases in carcinogenesis. Microsc Res Tech 59: 58-67, 2002.

23. Frumento G, Piazza T, Di Carlo E and Ferrini S: Targeting tumor-related immunosuppression for cancer immunotherapy. Endocr Metab Immune Disord Drug Targets 6: 233-237, 2006.

24. Kim R, Emi M and Tanabe K: Functional roles of immature dendritic cells in impaired immunity of solid tumour and their targeted strategies for provoking tumour immunity. Clin Exp Immunol 146: 189-196, 2006.
25. Cichon T, Jamrozy L, Glogowska J, Missol-Kolka E and Szala S: Electrotransfer of gene encoding Endostatin into normal and neoplastic mouse tissues: inhibition of primary tumor growth and metastatic spread. Cancer Gene Ther 9: 771-777, 2002.

26. Moser TL, Stack MS, Asplin I, Enghild JJ, Hojrup P, Everitt L, Hubchak S, Schnaper HW and Pizzo SV: Angiostatin binds ATP synthase on the surface of human endothelial cells. Proc Natl Acad Sci USA 96: 2811-2816, 1999.

27. Persano L, Crescenzi M and Indraccolo S: Anti-angiogenic gene therapy of cancer: current status and future prospects. Mol Aspects Med 28: 87-114, 2007. 